\title{
Resolved Mid-Infrared Imaging of AGN: An Isotropic Measure of Intrinsic Power
}

\section{P. Gandhi ${ }^{1}$, H. Horst ${ }^{2,3,4}$, A. Smette ${ }^{4}$, S. F. Hönig ${ }^{3}$, A. Comastri ${ }^{5}$, R. Gilli ${ }^{5}$, C. Vignali ${ }^{6}$, and W. J. Duschl ${ }^{2}$}

${ }^{1}$ RIKEN Cosmic Radiation Lab, 2-1 Hirosawa, Wakoshi, Saitama 351-0198, Japan Email: pg@crab.riken.jp

${ }^{2}$ Institut für Theoretische Physik und Astrophysik, Christian-Albrechts-Universität zu Kiel, Leibnizstr. 15, 24098 Kiel, Germany

${ }^{3}$ Max-Planck-Institut für Radioastronomie, Auf dem Hügel 69, 53121 Bonn, Germany

${ }^{4}$ European Southern Observatory, Alonso de Cordova 3107, Casilla 19001, Santiago, Chile

${ }^{5}$ Istituto Nazionale di Astrofisica (INAF) - Osservatorio Astronomico di Bologna, v ia Ranzani 1, 40127 Bologna, Italy

${ }^{6}$ Dipartimento di Astronomia, Università degli Studi di Bologna, Via Ranzani 1, I-40127 Bologna, Italy

Keywords. galaxies: active, galaxies: nuclei, infrared: galaxies, X-rays: galaxies, techniques: high angular resolution

We present a strong correlation between $12 \mu \mathrm{m}$ mid-IR and intrinsic X-ray $(2-10 \mathrm{keV})$ luminosities of local Seyferts. This work is based on new diffraction-limited mid-IR observations with the 8-m Very Large Telescope (VLT), resulting in the least-contaminated core fluxes of 42 Seyferts to date. Unobscured, obscured and Compton-thick sources all closely follow the same correlation,

$$
L_{\mathrm{MIR}} \propto L_{\mathrm{X}-\mathrm{ray}}^{1.11 \pm 0.07}
$$

which is tight enough to significantly constrain any residual star-formation within $\sim 70 \mathrm{pc}$ of the unresolved cores of active galactic nuclei (AGN). The mean $2 \mathrm{keV}$ to $12.3 \mu \mathrm{m} \mathrm{AGN} \mathrm{spectral}$ index is found to be $\alpha_{\mathrm{IX}}=-1.10 \pm 0.01$, largely independent of luminosity. The observed 12 $\mu \mathrm{m}$ bolometric corrections range between $\sim 10$ and $\sim 30$ if a known luminosity trend of intrinsic $\mathrm{X}$-ray bolometric corrections is assumed. Comparison with Infrared Space Observatory (ISO) data spanning a larger luminosity range suggests that our correlation can be extended into the quasar regime. Uncontaminated mid-IR continuum imaging is thus found to be an accurate proxy for the intrinsic power of an AGN, and we discuss the resulting implications for smooth and clumpy dust tori models as well as searches for any underlying non-thermal infrared emission components.

\section{References}

Gandhi, P., Horst, H., Smette, A., Hönig, S. F., Comastri, A., Gilli, R., Vignali, C., \& Duschl, W. J. 2009, A\&A, 502, 457 